\title{
The star formation history in circumnuclear regions of galaxies
}

\author{
Johan H. Knapen ${ }^{1,2}$, Emma L. Allard ${ }^{2}$, Marc Sarzi ${ }^{2}$, \\ Reynier F. Peletier ${ }^{3}$ and Lisa M. Mazzuca ${ }^{4}$ \\ ${ }^{1}$ Instituto de Astrofísica de Canarias, E-38200 La Laguna, Spain \\ ${ }^{2}$ University of Hertfordshire, Hatfield, AL10 9AB, UK \\ ${ }^{3}$ Kapteyn Astronomical Institute, 9700 AV Groningen, The Netherlands \\ ${ }^{4}$ NASA Goddard Space Flight Center, Greenbelt, MD 20771, USA
}

\begin{abstract}
Massive star formation in the central regions of spiral galaxies plays an important role in the dynamical and secular evolution of their hosts. Here, we summarise a number of recent investigations of the star formation history and the physical conditions of the gas in circumnuclear regions, to illustrate not only the detailed results one can achieve, but also the potential of using state-of-the-art spectroscopic and analysis techniques in researching the central regions of galaxies in general. We review how the star formation history of nuclear rings confirms that they are long-lived and stable configurations. Gas flows in from the disk, through the bar, and into the ring, where successive episodes of massive star formation occur. A detailed analysis of the ring in NGC 7742, where we use similar data to determine the physical conditions of the line emitting gas using a combination of ionisation and stellar population modelling, is described in a second poster paper (these proceedings, p. 000; Mazzuca et al. 2006).
\end{abstract}

Keywords. galaxies: evolution, galaxies: individual (NGC 4321, M100), galaxies: ISM, galaxies: kinematics and dynamics, galaxies: nuclei, galaxies: spiral, galaxies: starburst

\section{Introduction}

Starbursts, defined as relatively short periods of enhanced massive star formation (SF) activity, are important events in the evolution of galaxies. They can:

- enhance the luminosity of the host galaxies, facilitating the detection of those at larger distances,

- transform significant quantities of gas into stars,

- cause metal injection into, and mixing of, the interstellar medium,

- and help the secular evolution of galaxies.

We focus here on a particular class of low-luminosity starbursts occurring in nuclear rings in spiral galaxies, which allow the detailed study of their SF histories and of the physical properties of the gas in the circumnuclear region. Nuclear rings are relatively common, recognisable through their current massive SF in $20 \%$ of all spiral galaxies, and mostly occur in barred galaxies (Knapen 2005).

\section{Results}

We show results based on SAURON (Bacon et al. 2001) data of the bar and circumnuclear region of the SABbc galaxy M100=NGC 4321, as described in more detail in Allard et al. $(2005,2006)$. The $\mathrm{H} \beta$ emission outlines the star-forming circumnuclear ring and the SF along the bar. Gas with relatively low velocity dispersion accompanies the $\mathrm{SF}$, but both the low-dispersion gas and the SF are offset from the dustlanes in the bar. 
This shows how low-dispersion, cool, gas flows in through the bar into the circumnuclear region, leading to massive $\mathrm{SF}$ in the nuclear ring (Allard et al. 2005).

An analysis of the absorption lines ratios (Allard et al. 2006) shows that whereas the bar and the nucleus of M100 can be fitted with a simple stellar population (SPP) of some 3 Gyr old, the data points in the ring cannot be fitted by an SPP model.

Composite models can reproduce the observations. A previous episode of SF which formed the bar and bulge ended some 3 Gyr ago, and our preferred scenario is one where the current burst of SF is one of a series of several bursts which have occurred regularly over the past 0.5 Gyr or so (Allard et al. 2006).

Our more recent analysis of long-slit spectra (obtained with the ISIS spectrograph at the William Herschel Telescope) of five further nuclear rings confirms the general applicability of these composite models: they can reproduce the range of absorption line values covered by the observations (Allard et al. 2007). As discussed in more detail by Allard et al., other scenarios, for instance one in which SF has occurred continuously for the past $500 \mathrm{Myr}$ or so, following a period of a few Gyr without major massive SF activity, cannot be excluded. For reasons mostly to do with our dynamical understanding of the origin of nuclear rings (e.g., Knapen et al. 1995), however, our multiple burst scenario is the preferred one for these nuclear rings in general (Allard et al. 2007).

\section{Conclusions}

There is a strong correlation between the $\mathrm{H} \beta$ emission and low gas dispersion both in the ring and in the bar of the spiral galaxy M100. The offset between the dustlanes, which delineate shocks in the gas flow, and the cold gas/SF shows how the shocks channel inflowing gas inwards until it reaches the inner Lindblad resonance(s), where it accumulates into a ring, confirming the numerical and phenomenological picture of bar-driven inflow of gas into a circumnuclear ring, feeding the massive SF there.

The stellar absorption line index analysis shows how an early episode of SF put in place the underlying bar/disk/bulge. The current burst of SF defining the star-forming nuclear rings in spiral galaxies is but the latest in a series of such bursts which have occurred repetitively over the past 0.5 Gyr or so. The rings are indeed dynamically stable entities, which over large timescales can transform disk gas into stars at rates of a few $M_{\odot} /$ year, albeit not continuously. These stars, over time, add stellar mass to the bulge and thus drive the secular evolution of these galaxies.

We have thus illustrated how 2-D integral-field spectroscopy can lead to a significantly enhanced understanding of the SF history of complicated composite systems such as those discussed here.

\section{References}

Allard, E. L., Peletier, R. F. \& Knapen, J. H. 2005, ApJ 633, L25

Allard, E. L., Knapen, J. H., Peletier, R. F. \& Sarzi, M. 2006, MNRAS 371, 1087

Allard, E. L., Sarzi, M., Knapen, J. H. \& Mazzuca, L. M. 2007, MNRAS, submitted

Bacon, R., et al. 2001, MNRAS, 326, 23

Knapen, J. H. 2005, A\& A 429, 141

Knapen, J. H., Beckman, J. E., Heller, C. H., Shlosman, I. \& de Jong, R. S. 1995, ApJ 454, 623

Mazzuca, L. M., Sarzi, M., Knapen, J. H., Veilleux, S. \& Swaters, R. 2006, ApJ 649, L77 